\title{
Molecular Modeling and Electron Transport in Polyethylene
}

\author{
Yang Wang ${ }^{1}$, Kai $\mathbf{W u}^{1}$, \\ ${ }^{1}$ State Key Lab. of Electrical Insulation and Power Equipment, \\ Xi'an Jiaotong University, \\ Xi'an, 710049, China \\ David Cubero ${ }^{2,1}$ \\ ${ }^{2}$ Departmento de Física Aplicada I, \\ Escuela Politécnica Superior, \\ Universidad de Sevilla, \\ Seville, 41011, Spain \\ and Nick Quirke ${ }^{3,1}$ \\ ${ }^{3}$ Department of Chemistry, \\ Imperial College, \\ London, SW7 2AZ, UK
}

\begin{abstract}
Polyethylene is commonly used as an insulator for AC power cables. However it is known to undergo chemical and physical change which can lead to dielectric breakdown. Despite almost eighty years of experimental characterization of its electrical properties, very little is known about the details of the electrical behaviour of this material at the molecular level. An understanding of the mechanisms of charge trapping and transport could help in the development of materials with better insulating properties required for the next generation of high voltage $\mathrm{AC}$ and DC cables. Molecular simulation techniques provide a unique tool with which to study dielectric processes at the atomic and electronic level. Here we summarise simulation methodologies which have been used to study the properties of $\mathrm{PE}$ at the molecular level, elucidating the role of morphology in the trapping of excess electrons. We find that polyethylene has localised states due to conformational trapping extending below the mobility edge (above which the excess electrons are delocalised), at $-0.1 \pm 0.1 \mathrm{eV}$ with respect to the vacuum level. These trap states with localisation lengths between 0.3 and

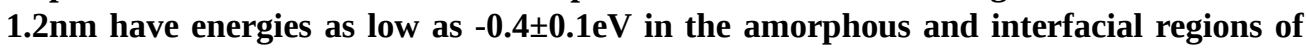
polyethylene with more positive values in lamella structures. Crystalline regions have a mobility edge at $+0.46 \pm 0.1 \mathrm{eV}$, so we would expect transport by electrons excited above the mobility edge to delocalised states to be predominantly through amorphous regions if they percolate the sample.
\end{abstract}

Index Terms - Molecular simulations, polyethylene, methodologies, electron trapping, electron transport, morphology.

\section{INTRODUCTION}

Polyethylene's low electric conductivity, low dielectric loss, high dielectric strength and outstanding mechanical properties [1] make it a common choice as an insulator for AC power cables. However, under certain conditions, polyethylene may suffer from chemical and electrical aging or degradation after long-standing operation under voltage, which may finally lead to dielectric breakdown [2].

A major contribution to aging and breakdown is thought to be made by trapped charges, called the 'space charge' [2]. The accumulation of space charge distorts the local electric field, facilitating phenomena such as treeing as well as generating chemical and physical defects through the local release of trapping energies (of the order of an $\mathrm{eV}$ ), eventually leading to the degradation of the insulation, although the exact mechanisms operating are still not fully understood. The formation and transportation mechanism of space charge has been the subject of extensive experimentation [2]. For example, by monitoring the threshold electric field for charge accumulation it has been found that charge injection is strongly affected by the material of the electrode [3-5]. Space charge formation and transport phenomena under DC stress can be observed using the Pulsed Electro-Acoustic (PEA) method [6-9]. This technique has been used to show that the interfaces either between multilayers of $\mathrm{PE}$ or between PE and the electrode, is prone to space charge accumulation, and thus might be considered to be weak points with respect to insulating properties [10-12]. 
Despite a vast literature concerned with the experimental characterization of its electrical properties, very little is known about the details of the electrical behavior of this material, especially at the molecular level. An understanding of the mechanisms of charge trapping and transport in these insulators and how such processes are affected by local physical structures and chemical impurities could help in the development of materials with better insulating properties required for the next generation of high voltage AC and DC cables. In what follows we summarize molecular simulation methodologies which have been used to study the properties of PE at the molecular level, elucidating the role of morphology as well as chemical imperfections and additives on the trapping of excess electrons.

The paper is organized as follows: Section II presents simulation methodologies which have been found useful in understanding PE, including: Molecular Dynamics (MD) for modeling morphology, Density Functional Theory (DFT) for calculating electronic properties using the generated morphologies, the Lanczos method which can be used to predict the density of states of excess electrons in a given PE structure, and the Surface Hopping method which predicts the quantum transitions between such states. Section III reviews current work focusing on the relationship between the electronic states and PE morphologies. The findings are discussed and some conclusions presented in Section IV.

\section{MOLECULAR METHODOLOGIES}

In many branches of science and engineering, molecular simulation techniques (including MD, DFT, etc.) are an integral part of modern research programs. They can provide a theoretical basis upon which to interpret experimental results as well as a tool for discovery in their own right. For example, computer simulations can explore the properties of 'perfect' materials free from chemical and morphological contamination [13]. Once the relationship between the simulated and experimental systems is established, the simulation can be used to explore the effects of systematically changing both external (pressure, temperature, density, etc.) and internal (composition, morphology, defect structure, etc.) variables. In this section, we provide a brief introduction to simulation techniques which have been found to be useful for polymeric materials.

\subsection{MOLECULAR DYNAMICS METHODS}

Molecular dynamics (MD) [13, 14] is widely used to simulate the trajectories of molecules or atoms in a classical many-body system by solving Newton's equations of motion. The thermodynamic, structural and transport properties of the system can then be obtained by taking time averages over the trajectories.

This method is widely used for almost any molecular system at moderate or high temperatures (such as at normal conditions, $300 \mathrm{~K})$, so that quantum effects in the dynamics of atoms or molecules can be safely neglected.

The interactions between the molecules are described using a force-field, that is, a set of potential energy functions. These functions are usually empirical, consisting of intra-molecular terms representing the energy associated with for example atom-atom bonds, and orientation (valence and dihedral angles) and intermolecular terms representing the van der Waals or dispersion interactions, Coulomb interactions between charges, directional bonds such as H-bonds and, where necessary, three or many-body terms.

Given the force-field and a set of initial conditions, the trajectory of the whole system is usually integrated as a function of time using a simple time-step algorithm such as the velocity Verlet method $[14,15]$. Clearly, it is important to choose a time-step small enough so that the generated trajectories are realistic. Typical time-steps for a polymeric system are of the order of a few femtoseconds and averages are taken over thousands to hundreds of thousands of configurations (time-steps) of the system.

The standard MD simulation generates a micro-canonical ensemble, with constant number of particles (N), volume (V) and energy (E). The instantaneous temperature $\mathrm{T}$ is obtained from the total kinetic energy as given by the equipartition theorem.

By introducing to the simulation a thermostat to fix the system temperature (popular choices are those given by Berenson, Andersen or Nose-Hoover) or a barostat to fix the pressure, MD simulations can be also carried out in a canonical ensemble (NVT) or an isothermal-isobaric ensemble (NPT) $[14,15]$.

When only equilibrium properties are required, Monte Carlo (MC) simulation [14] provides an alternative methodology. MC does not generate realistic trajectories but samples configuration space using Metropolis sampling to generate a set of configurations consistent with the statistical mechanical probability distribution for the given ensemble (i.e. isothermalisobaric corresponding to constant number of atoms $\mathrm{N}$, pressure $\mathrm{P}$, and temperature $\mathrm{T}$ or grand canonical corresponding to constant chemical potential $\mu$, volume $\mathrm{V}$, and temperature $\mathrm{T}$.). The fictitious trajectories are generated by randomly changing the microscopic degrees of freedom (atom coordinates, etc). The name of the method comes from the famous Monte Carlo casino, due to the extensive use of random numbers. Thermal equilibrium is usually guaranteed by implementing an ergodic sampling that also complies with detailed balance.

Thus far we have only considered classical methods where the electronic degrees of freedom are incorporated into the potential function describing the inter-atomic interaction. For explicit electronic properties, such as the density of electron states, or excess electron dynamics, a quantum mechanical simulation method is required.

\subsection{DENSITY FUNCTIONAL THEORY}

Density Functional Theory (DFT) (see book eg. reference [16]) is a very powerful and successful method for investigating the electronic structure of a many-body system (i.e. a multi-electron system). In many cases the results of DFT calculations for solid-state systems agree very well with experimental data. For covalent, metallic and ionic bonds DFT will usually be within 2-3\% for the geometry (bond 
lengths, cell parameters), and $0.2 \mathrm{eV}$ for the bonding energies (GGA, see below) [17].

The starting point of the theory is the Born-Oppenheimer adiabatic approximation, in which the nuclear and electronic degrees of freedom are separated. The electrons are considered to adjust almost instantaneously to a change in nuclear coordinates which can be considered fixed whilst the system is solved for the electronic states.

DFT is based on the Hohenberg-Kohn (HK) theorems [16, 18]. The first one states that "The ground-state energy from the Schrödinger equation is a unique functional of the electron density", which implies that the ground-state electron density $n(r)$ uniquely determines all properties, including the energy and wave function, of the ground state. The second HK theorem defines an important property of the functional: "The electron density that minimizes the energy of the overall functional is the true electron density corresponding to the full solution of the Schrödinger equation". Therefore, by minimizing the functional of the electron density, the electronic structure of the system can be then calculated.

The Schrödinger equation of the many electron system can be rewritten as a set of single-particle equations for an auxiliary non-interacting system, the Kohn-Sham (KS) equation [19],

$\left[\frac{\mathrm{h}^{2}}{2 \mathrm{~m}} \nabla^{2}+V(r)+V_{H}(r)+V_{X C}(r \psi] r \quad \neq \varepsilon \psi r \quad i_{i}(1)(1)\right.$

The terms on the left-hand side of this equation are, in order, the kinetic energy; the Coulomb interactions between the electron and the nuclei; the Hartree term describing the electron-electron Coulomb repulsion; and the exchangecorrelation potential which includes all the many-particle interactions. This latter term can be defined as a functional derivative of the exchange-correlation energy as,

$V_{X C}(r)=\frac{\partial E_{X C}(r)}{\partial n(r)}$

where $n(r)$ refers to the electron density. If the exchangecorrelation energy is known as a functional of the density, we have a closed set of self-consistent equations yielding a solution to the electronic structure problem.

Since the Hartree term $V_{h}(r)$ and $V_{x c}(R)$ depend on $n(r)$, which depends on the KS wave-functions $\psi_{\mathrm{i}}(\mathrm{r})$ which in turn depend on the total effective potential $\mathrm{V}(\mathrm{r})+\mathrm{V}_{\mathrm{h}}(\mathrm{r})+\mathrm{V}_{\mathrm{xc}}(\mathrm{R})$, the problem of solving the Kohn-Sham equation has to be done in a self-consistent way. Starting with an initial guess for $\mathrm{n}(\mathrm{r})$, the corresponding effective potential can be calculated and the Kohn-Sham equations solved for $\psi_{\mathrm{i}}(\mathrm{r})$. From these a new density is calculated and the process starts again. This procedure is then repeated until convergence is reached.

The exact form of the exchange-correlation energy functional is only known for the free electron gas. Thus, in practice, $\mathrm{E}_{\mathrm{xc}}$ is calculated using approximations, such as local density approximation (LDA) [19], or generalized gradient approximations (GGA) [17], or B3LYP [20], see also [21]. Finding the right functional can be considered the equivalent problem to finding the potential function in a classical simulation.

The Kohn-Sham wave functions are also sometimes identified with electronic states, and the KS energies are taken as estimates of single-particle energies, including that of an excess electron or a hole in the system. While this is not a rigorous procedure, this identification provides a simple method to obtain estimates in many systems that would be very difficult to obtain otherwise. Note however that the results of this uncontrolled approximation are not expected to be as accurate as the standard results (total energy, total electronic density, plus the atomic positions obtained when used for equilibration or dynamics) of DFT.

Within the framework of the Born-Oppenheimer adiabatic approximation, an ab initio Molecular Dynamics technique can be obtained when the forces exerted by the electrons on the nuclei are computed using DFT at every time-step. The nuclei are then moved using Newton's laws as in the standard MD method. One of such approaches is the so-called CarParrinello method [22]. As an artefact to speed up the calculations, the Car-Parrinello method explicitly introduces the electronic degrees of freedom as fictitious variables. The resulting fictitious dynamics keeps the electrons on the electronic ground state, thus avoiding an explicit electronic minimization at each time-step. From among all ab-initio Molecular Dynamics techniques, the Car-Parrinello method is perhaps the most widely used.

Finally, let us note that DFT calculations do not provide fully $a b$ initio solutions of the full Schrödinger equation because the exact functional is not known, however, approximate functionals can be tuned to experimental data if sufficient information is available for the system of interest. Among the shortcomings of DFT is its inability to account accurately for the weak van der Waals attractions that exist between atoms and molecules (see [23] for a summary of current progress). In addition, being strictly a ground state theory standard DFT cannot predict excited states (however Time-dependent density functional theory [24] can predict the properties of excited states of a multi-electron system) and is not capable of predicting accurate band gaps for semiconducting and insulating materials. However many variations and elaborations of density functional theory codes exist which address these problems (see for example [25, 26] for the successful prediction of band gaps), and in general the method is extremely powerful.

In the following, we conclude this section by discussing two methods which allow quantum calculations of a single electron in the system. These calculations are appropriate for the study of excess electrons in the dielectric, when the number of them is not too large, so that we can treat the excess electrons as independent particles. These methods are more amenable when compared to DFT, requiring much less 
computer power and without many of the typical difficulties of the DFT techniques.

\subsection{LANCZOS METHOD}

The Lanczos algorithm is a numerical method for diagonalising (i.e. finding the eigenstates and eigenfunctions) for a given matrix A. Here the matrix of interest is the Hamitonian $\mathrm{H}_{\mathrm{e}}$ describing the interaction of an excess electron with PE through a pseudopotential $\mathrm{V}(\mathrm{x}, \mathrm{y}, \mathrm{z})$, which is an input to the calculation (for details see [27] and references therein). The Schrodinger equation is then solved for the ground and excited states of the excess electron (a single electron) for given configurations of atoms, these corresponding to different morphologies. The Lanczos method is particularly useful for the purpose of solving the Schrodinger equation for a single electron on a three dimensional grid of points in real space $(x, y, z)$, because the Hamiltonian is sparse, having many negligible terms. It is based on the generation of a set (or a set of blocks) of basis functions which are designed to allow the efficient diagonalisation of $\mathrm{H}_{\mathrm{e}}$, so that the ground and low lying excited states of the excess electron are found. This calculation directly provides the information required to estimate the density of excess electron states of our system. This information can then be used to calculate transport properties such as excess electron mobilities due to extended states.

We briefly describe here the Block Lanczos method proposed by Webster, Rossky and Friesner [28, 29]. We start from a trial set of $\mathrm{n}$ basis functions $\left\{\psi_{\mathrm{n}}{ }^{1}\right\}$ comprising the vectors $\psi_{\mathrm{i}}$ (for example sine or cosine functions of position). The operator $\mathrm{W}=\exp \left(-\beta \mathrm{H}_{\mathrm{e}}\right)$, where $\beta$ is a positive constant, is applied to this set to get a second set of eigenfunctions $\left\{\psi_{\mathrm{n}}^{2}\right\}$. In each step the Gram-Schmidt method [30] is used to guarantee that this new set is orthogonal to the first set and the basis functions in a given set are kept orthogonal between themselves. The first set $\left\{\psi_{\mathrm{n}}{ }^{1}\right\}$ is the first 'block' of our space, (called the Krylov space), with which we diagonalise W. The set $\left\{\psi_{\mathrm{n}}{ }^{2}\right\}$ is the second block, and so on. By repeated application of the operator $\mathrm{W}$, we can obtain $\mathrm{N}_{B}$ blocks, which constitute the space within which the operator $\mathrm{W}$ is diagonalised. $\mathrm{W}$ has the same eigenvalues and eigenfunctions as $\mathrm{H}_{\mathrm{e}}$ and is used because it increases the convergence of the solution towards the ground and lower excited states of the system, which are our primary interest. The results depend on the parameter $\beta$, which is varied until the solutions converge.

\subsection{SURFACE HOPPING}

The Lanczos method provides the excess electron properties for a single atomic configuration. The surface hopping technique (see reference [31]) is a method to simulate the dynamics of a quantum particle (here to be identified with an excess electron) in interaction with a larger classical system (such as PE), which allows us to go beyond the Born-Oppenheimer adiabatic approximation. We describe here the algorithm proposed by Tully [32, 33], which accounts approximately for the quantum transitions between energy levels (surfaces) and the way these transitions (hopping) affect the dynamics of atoms in the system, which are treated classically. The term 'surface' here refers to the single electron energy eigenstates of the system.

Let $r=(x, y, z)$ refer to the coordinates of the quantum particle and $\mathrm{R}(\mathrm{t})=\left\{\mathrm{R}_{\mathrm{i}}(\mathrm{t})\right\}$ those of the classical molecules, then the total Hamiltonian describing the electronic motion, $\mathrm{H}_{\mathrm{e}}(\mathrm{r}, \mathrm{R})$, will be a time-dependent operator. The wave function of our quantum particle $\psi(\mathrm{r}, \mathrm{t})$ can be solved using the timedependent Schrödinger equation,

ih $\frac{\partial \psi}{\partial t}=H \psi$

It is helpful to use a set of adiabatic basis functions $\Phi_{\mathrm{n}}(\mathrm{r}$, $\mathrm{R})$, which can be computed using the Lanczos algorithm described in the previous Section,

$\psi(r, t)=\sum_{n} a_{n}(t) \Phi_{n}(r, R(t))$

Where the $\mathrm{a}_{\mathrm{n}}(\mathrm{t})$ are expansion coefficients. Substituting (4) into (3), multiplying from the left by $\Phi_{\mathrm{m}}$ and integrating over $r$ gives

$\frac{d a_{m}}{d t}=\sum_{n} d_{m n} a_{n}$

ere

$$
\begin{aligned}
& d_{m n}=-i E_{n} \delta_{n m} / \mathrm{h}-<\phi_{m}|\partial / \partial t| \phi_{n}> \\
& <\phi_{m}|\partial / \partial t| \phi_{n}>=\sum_{j} \frac{d R_{j}}{d t} \bullet D_{m n}^{j} \\
& D_{m n}^{j}=<\phi_{m}\left|\nabla_{j}\right| \phi_{n}>
\end{aligned}
$$

By rewriting Eq. (5) in the equivalent density matrix notation, we define $\rho_{n m}=a_{m}{ }^{*} a_{n}$, so that the Schrödinger equation can be written as

$$
\frac{d \rho_{m n}}{d t}=-\sum_{m} 2 \operatorname{Re}\left(d \rho_{n n}{ }_{m n}\right)
$$

The diagonal elements $\rho_{\mathrm{nn}}$ are the electronic state population and the off-diagonal elements $\rho_{n m}$ define the coherence. Each term in the sum can be interpreted as the number of transitions from the state $n$ to the state $m$ per unit time. Therefore, during a small time step $\delta$ we can express this quantity as the probability that the electron is in state $n$ multiplied to the probability of the transition to other state: 


$$
2 \operatorname{Re}\left(d_{R n} \delta=R \phi \quad n n \quad n \rightarrow m\right.
$$

Once a transition to other state has been decided by using Monte Carlo transition probability [14], we must change the kinetic energy of the classical subsystem in order to conserve energy. This is usually done by scaling the velocities $\mathrm{v}_{\mathrm{j}}$ of the classical particles in the direction of the corresponding nonadiabatic coupling vector $\mathrm{D}_{\mathrm{mn}}^{\mathrm{j}}(\mathrm{t})$.

In this implementation of the surface hopping method, the classical particles are moved at fixed intervals according to MD. Whether there is a quantum transition is decided during the last time step (i.e. if $\mathrm{t}$ is the current time step, the transition is decided in the interval from $t-\Delta$ to $t$.). If the hop is accepted we scale the atom's velocities at $t$. The quantum force over the atom $\mathrm{j}$ can be derived from the HelmanFeyman theorem as

$$
F_{j}=-\left\langle\phi_{n}\left|\nabla_{j} H_{e}\right| \phi_{n}\right\rangle
$$

This implementation of surface hopping method makes it possible to study the non-adiabatic dynamics of a small quantum system in contact with a larger classical system in an approximate way. As such, it goes beyond the scope of DFT or the Car-Parrinello method, since these latter techniques assume that the whole system always remains in the ground-state.

\section{Molecular modelling of Polyethylene}

\subsection{PE MORPHOLOGIES AND ITS CONFORMATIONAL DISORDERS}

Early simulation studies on bulk PE used n-alkane short chains to mimic long chain PE. Simulations of PE chains, each with $50 \mathrm{CH}_{2}$ units, below and above the glass transition temperature, was performed to study $\mathrm{PE}$ physical and conformational properties such as chain conformation, glass transition or free-volume distribution [34-37]. The transition between rotational isomers in $\mathrm{PE}$ chains of $100 \mathrm{CH}_{2}$ units was analyzed in [38]. All-trans PE chains of $60 \mathrm{CH}_{2}$ units was studied [39] around the melting point. However, none of those simulations observed the chain folding phenomenon widely seen in experiments, and believed to be one of the basic motifs of the PE bulk sample.

Quantum effects on an assumed orthorhombic phase of crystalline PE were studied in reference [40] by means of path-integral Monte Carlo (PIMC) simulations. The low temperature range, from $25 \mathrm{~K}$ to $300 \mathrm{~K}$, was explored in the NPT ensemble by looking at PE chains of two lengths, $C_{12}$ and $\mathrm{C}_{24}$ (with a total number of atoms of 432 and 864). Structural parameters such as the lattice constants, bond lengths, and bond angles and their dependence with temperature were analyzed, showing their reduction as the temperature is decreased.

By performing MD simulations of $\mathrm{PE}$ chains of various lengths, using a united-atom model with torsional barriers of 2, 3 and $6 \mathrm{Kcal} / \mathrm{mol}$, with the remaining parameters being taken directly from the Dreiding forcefield, the chain folding phenomenon was observed [41, 42]. They found that during simulations with a torsional barrier of $2 \mathrm{kcal} / \mathrm{mol}$, PE chain folding occurs when the chains have more than $150 \mathrm{CH}_{2}$ units, long enough that the average inter-molecular van der Waals energy is low enough to compensate the increase in energy owing to torsion angle and bond angle deformation in the folds. The reported behaviour was in good agreement with the experimental observations [43].

More recently, multi-phase polyethylene morphologies including crystalline, amorphous, lamellae and interfacial regions have been studied using MD [44, 45]. As discussed in reference [2], a polyethylene sample may contain amorphous, lamellae and crystalline regions of various sizes depending on the material processing details. In reference [44], model crystalline and lamellae regions were created by geometry optimization using the all-atom COMPASS forcefield in Materials Studio 5.5 [46]. For the lamellae regions, gauche and anti-gauche defects were introduced into ten all-trans PE chains with $552 \mathrm{CH}_{2}$ units in order to enable them to fold back and forth upon themselves, resulting in a lamellar thickness of around $5.0 \mathrm{~nm}$. The structures were then imported into LAMMPS simulation package [47], and equilibrated using a united-atom force field [48] optimized for long chain n-alkanes. The amorphous and interfacial regions were prepared by melting the corresponding lamellae blocks either in NPT or N $\mu$ T ensembles with a time-varying thermostat in LAMMPS. The resulting morphologies were in good agreement with experiment.

As observed experimentally [49], polyethylene contains a significant number of nanometre sized voids. In order to explore their influence on excess electrons, nanometre sized voids were produced in the simulated amorphous phase by expanding a test particle [44]. By calculating the Gibbs free energy to create different radius of spherical voids in amorphous PE, a surface tension of $36 \mathrm{mN} / \mathrm{M}$ was obtained, in good agreement with the experimental value of $35.7 \mathrm{mN} / \mathrm{M}$ at $20^{\circ} \mathrm{C}$ for linear HDPE [50].

\subsection{ELECTRONIC PROPERTIES OF PE}

Surface trapping states of PE was studied [51] using DFT in the gradient-corrected local density approximation (LDA), supplemented with empirical long-range tails in order to properly account for van der Waals forces. Two PE surfaces were considered, representative of two classes of surfaces that differ in the orientation of the PE chains with respect to the planar surface i.e. with the chains oriented either parallel or perpendicular to the surface vector. A negative electronaffinity was found, with values of $-0.17 \mathrm{eV}$ and $-0.10 \mathrm{eV}$ for surfaces with chains perpendicular and parallel to the surface, respectively. Negative electron-affinities are in agreement with experiment and other simulation results (see below), though in this DFT study the analysis is based on the identification of KS eigenvalues (section 2) with singleparticle energy levels.

Dynamical DFT simulations have also been performed [52] within the framework of the Car-Parrinello (CP) technique. Calculations were carried out with an injected electron neutralized by a background charge in a crystalline cell with four PE chains (each of them with $7 \mathrm{CH}_{2}$ units), starting from an equilibrium configuration. A similar calculation was also 
performed with an injected hole. A self-trapped state for the injected electron was found, localizing near an inter-chain area which involved a pair of trans-gauche defects. The hole was observed to remain delocalized throughout the simulation cell, being of an intra-chain nature.

In reference [53], an electron-hole pair, an exciton, was injected in a similar crystalline cell, being observed to be long-lived, displaying no apparent direct channel for nonradiactive recombination. In contrast, very recent DFT calculations [54] have found a direct channel for the recombination of the exciton via the breaking of a $\mathrm{C}-\mathrm{H}$ bond. The discrepancy with the results in [53] might be due to the exchange-correlation functional employed or the different methods chosen to improve the deficiencies of standard DFT. However, reference [54] presents a convincing scenario in which the recombination of an electron-hole pair under electrical stress could lead to degradation of PE.

Research focused on the electron trapping density of states identifying both physical and chemical defects were also carried out. To model the physical defects [55], MD simulations of amorphous tridecane $\left(\mathrm{n}-\mathrm{C}_{13} \mathrm{H}_{28}\right)$ were used as a model of short sections of $\mathrm{PE}$ to generate localized conformational defects. The trap energy was defined as the difference between the electron affinity of the wax molecule with and without conformational defects (i.e. not all trans) as $\mathrm{E}_{\mathrm{t}}=\mathrm{EA}\left(\mathrm{C}_{13} \mathrm{H}_{28}\right)-\mathrm{EA}\left(\mathrm{n}-\mathrm{C}_{13} \mathrm{H}_{28}\right)_{\text {all-trans. The electron affinity was }}$ obtained from DFT as implemented in the code DMol [56]. A similar methodology was used to estimate trapping energies for chemical defects and an approximate excess electron density of states obtained. Other workers have used similar methods [57-59]. Some results for chemical defects are given in table 1 , clearly there are some very deep traps $(>0.5 \mathrm{eV})$ but also some shallow traps $(<0.5 \mathrm{eV})$ which overlap in energy with those caused by conformational defects.

Table 1. Trap depths of some chemical impurities and docomposition

\begin{tabular}{c|c}
\multicolumn{2}{c}{ products in PE [57] } \\
\hline Molecule & $\begin{array}{c}\text { Trap depth } \\
(\mathrm{eV})\end{array}$ \\
\hline $\begin{array}{c}\text { 5-decanone } \\
\left(\mathrm{C}_{10} \mathrm{H}_{20} \mathrm{O}\right)\end{array}$ & -0.453 \\
\hline 5 -vinyl \\
$\left(\mathrm{C}_{10} \mathrm{H}_{20}\right)$ & -0.122 \\
\hline $5-$ decanol \\
$\left(\mathrm{C}_{10} \mathrm{H}_{21} \mathrm{O}\right)$ & -0.186 \\
\hline $5-$ decanal \\
$\left(\mathrm{C}_{10} \mathrm{H}_{20} \mathrm{O}\right)$ & -0.445 \\
\hline $\begin{array}{c}\text { Cumylalchohol } \\
\left(\mathrm{C}_{9} \mathrm{H}_{12} \mathrm{O}\right)\end{array}$ & -0.28 \\
\hline $\begin{array}{c}\text { Acetophenone } \\
\left(\mathrm{C}_{8} \mathrm{H}_{8} \mathrm{O}\right)\end{array}$ & -0.9 \\
\hline Alpha-methylstyrene \\
$\mathrm{C}_{9} \mathrm{H}_{10}$ & -1.53 \\
\hline Cumene & \\
\hline$\left(\mathrm{C}_{9} \mathrm{H}_{12}\right)$ & -0.04 \\
\hline
\end{tabular}

A more detailed study of the physical defects in amorphous and crystalline PE and their effect on excess electrons was carried out $[27,60]$ using Lanczos method to compute the excess electronic states of static configurations taken from
MD simulations (for ethane, methane, propane, crystalline $\mathrm{PE}$, and amorphous PE using a single chain with $300 \mathrm{CH}_{2}$ units). The pseudo-potential, describing the interaction of the excess electron with the atoms, was adjusted to fit the experimental data for the threshold of conduction in fluid ethane and propane. It contained short-range repulsive potentials, and an attractive part, which accounts for the polarization interaction between the excess electron and the dielectric, based on multi-centre polarizabilities obtained by fully ab initio methods. The amorphous PE samples showed Anderson localization [61], with a mobility edge, separating localized and delocalized states. The electronic density of states (DoS) and the electron mobility at the amorphous phase was calculated using the Kubo-Greenwood equation [62]. Further work [63], using non-adiabatic simulations of an excess electron in amorphous PE, permitting the deformation of the material due to the presence of the electron, showed the spontaneous formation of localized small polaron states in which the electron is confined. Despite allowing nonadiabatic transitions by using Tully's surface hopping algorithm [64], the simulations showed mainly adiabatic dynamics.

Very recently, the analysis of references [27, 60] has been extended to larger systems and more representative regions of $\mathrm{PE}$, including lamellae and interfaces between lamellae and amorphous [44, 45]. A clear correlation between local atomic density (calculated as the number of $\mathrm{CH}_{2}$ united atoms per unit volume in a cube of side $0.5 \mathrm{~nm}$ at a given position) and electron probability has been found in all PE phases, showing that the electron is more likely to localize in lower density regions.

Simple visual inspection of the localized states showed that the electron is sitting at regions with a reduced local atomic density, as illustrated in Figure 1. In the interfaces between lamellae and amorphous phases, the lowest atomic density values are found either in the amorphous regions or near the interface [45]. Figure 2 shows a typical ground state localized in that area.

In order to make this association more quantitative, we have calculated the Pearson correlation coefficient between the probability density of the excess electron and the local PE atomic density [45]. This dimensionless coefficient is usually defined as

$$
r=\frac{\sum_{i=1}^{n}\left(X_{i}-\bar{X}\right)\left(Y_{i}-\bar{Y}\right)}{\sqrt{\sum_{i=1}^{n}\left(X_{i}-\bar{X}\right)^{2}} \sqrt{\sum_{i=1}^{n}\left(Y_{i}-\bar{Y}\right)^{2}}}
$$

where $\mathrm{X}$ and $\mathrm{Y}$ are the variables of interest, here to be identified with the electronic probability density (the square of the wave function) in a given electronic state and the local atomic density. The index $\mathrm{i}$ in (12) runs through the 3D grid points in the system. The coefficient $r$ measures the strength of the linear association between both variables: a value of zero indicates no correlation, while nonzero values show correlation or anti-correlation. In the amorphous phase, starting with the ground state, the Pearson correlation shows a significant anti-correlation value of about -0.4 in regions of the material where the electron has a probability of $90 \%$. The 
next first 10 excited states, still below the mobility edge, show a correlation of about -0.2 , while for the first 10 states above the mobility edge the correlation is reduced to -0.1 , which is still significant, especially taking into account that these latter states are extended.

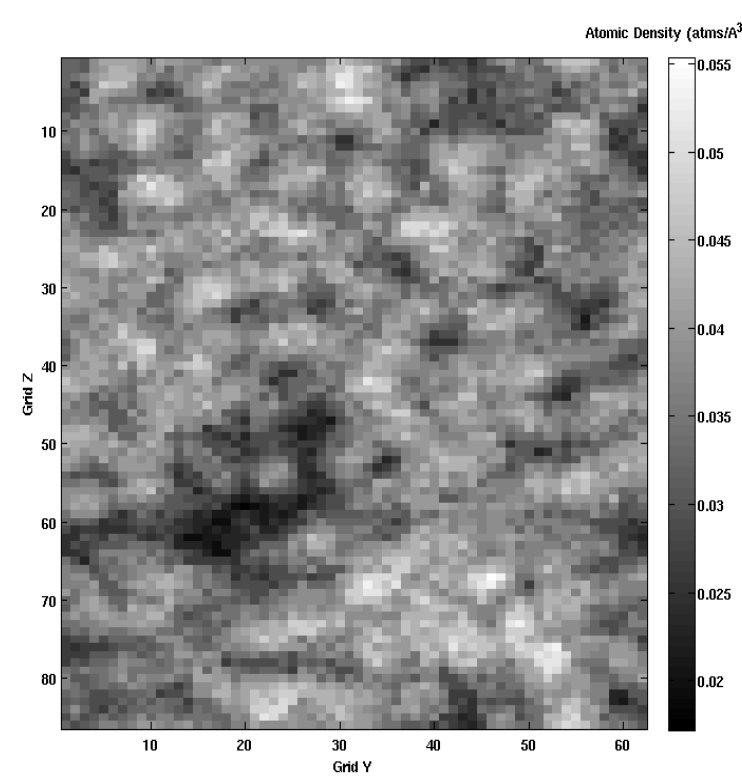

(a) Atomic Density

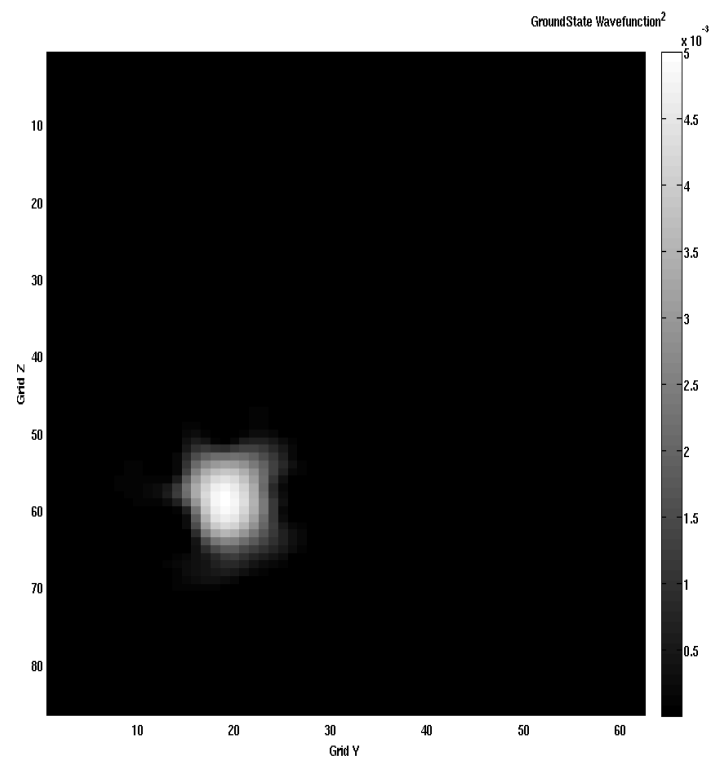

(b) Electronic Probability Density for Ground State

Figure 1. Slab sections showing the atomic density (upside) and the electronic probability density (downside) in the ground state for amorphous PE [45].

Another way to look at this correlation is presented in reference [44]. First, the free volume or cavity number distribution in the system is computed after identifying cavities with local minima of the atomic density. A radius is assigned to each cavity based on the radial distribution function from the centre of the cavity. Then, each localized state is associated with a cavity, providing an average energy and localization length as a function of the cavity radius. The cavity number distribution can be then mapped to produce a prediction of the density of states (DoS) based on the free volume distribution. This prediction is shown [44] to accurately predict the observed DoS in the three PE bulk

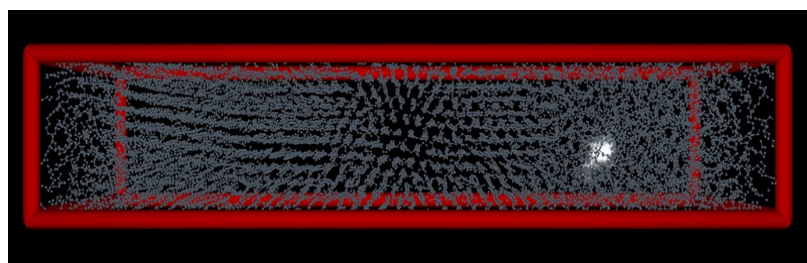

Figure 2. Typical interface configuration with lamellar and amorphous regions at room temperature and the corresponding excess-electron ground state. The lamellar structure can be seen in the first 3/4 part of the simulation cell. The electronic probability density is represented as a source of light, the lighter the point, the higher the probability.

phases studied- amorphous, lamellae and crystalline, when a correction based on the localization length is carried out. The correction is needed because localized states with higher energy values are not restricted to single cavities, instead extending through adjacent ones.

\section{Discussion AND CONCLUSIONS}

As we have seen, molecular modeling techniques have enabled some progress to be made in understanding the fundamentals of charge trapping and electron transport in polyethylene. Previous work has identified physical, (conformational) [52, 55] and chemical (impurities and decomposition products) [57-59] electron traps in models of polyethylene and characterized them using ab initio methods. Some of this information was used to create a preliminary distribution function representing the density of trap states (DoS) as a function of electron energy and employed in a Monte Carlo simulation to predict the current-voltage [65] characteristics of model polyethylene, showing how once the DoS is known the electrical properties can be estimated. A more complete treatment of conformational trapping using the fast Fourier transform block Lanczos diagonalization algorithm, described in section 2, has resulted in densities of states for the different morphologies found in polyethylene $[44,45]$. A simple theory based on this work now links the physical traps in polyethylene to nanovoids associated with regions of low density in the material.

Our current understanding of conformational excess electron trapping based on molecular modeling [44] has localized states extending below the mobility edge (above which the electrons are delocalized), at $-0.1 \pm 0.1 \mathrm{eV}$ with respect to the vacuum level. These trap states with localization lengths between 0.3 and $1.2 \mathrm{~nm}$ have energies as low as $-0.4 \pm 0.1 \mathrm{eV}$ in the amorphous and interfacial regions of polyethylene with more positive values in lamella structures. Note crystalline regions have a mobility edge at +0.46 $\pm 0.1 \mathrm{eV}$, so we would expect transport by excess electrons excited above the mobility edge to delocalized states to be predominantly through amorphous regions if they percolate the sample. 
At low electron concentrations, transport above the mobility edge will be dominated by the filling of the deepest traps which are likely to be chemical traps $\sim-1 \mathrm{eV}$. As the deepest traps are filled the excess electron mobility will increase dramatically towards a value that corresponds to multiple trapping between more shallow conformational traps [65].

Nonadiabatic simulations (Section 2) of an excess electron in amorphous $\mathrm{PE}$ at room temperature showed the spontaneous formation of localized small polaron states in which the electron was confined in a spherically shaped region with a typical localization length of $0.5 \mathrm{~nm}$. The selftrapping energy was $\sim-0.06 \pm 0.03 \mathrm{eV}$, with a lifetime on the time scale of a few tens of picoseconds. The smallness of the self-trapping energy is consistent with an adiabatic hopping mechanism assisted by phonons, as observed in the simulations. The contribution to the mobility due to hopping between these self-trapped states may well be of same order of magnitude as the mobility due to excited electrons above the mobility edge.

The model studies confirm the picture of deep chemical and shallow conformational electron traps current in [2]. They also however provide the means to go further and begin to investigate the influence of trapped electrons, the space charge, on the surrounding material. For example there has been very little work on the fate of the energy given up by trapped electrons in polyethylene (but see [58] for trapped excitons) which may alter the environment leading to local damage and ageing. The very recent DFT results [54] suggesting PE degradation by exciton recombination provide an interesting scenario that needs to be confirmed. In this regard, though molecular modeling studies provide a great insight in the understanding of polymeric dielectrics, these studies need to be assisted by experiments on very pure polymeric materials (including polyethylene) in which the effects of chemical traps from additive chemicals and/or radiation damage on electrical properties can be separated from those of physical traps related to the various polymer morphologies present in real materials.

\section{ACKNOWLEDGMENT}

The authors wish to thank the funding support from State Key Lab. of Electrical Insulation and Power Equipment under Project EIPE13203.

\section{References}

[1] C. C. Ku and R Liepins, "Electrical properties of polymers", Hanser publishers, 1987

[2] L. A. Dissado and J. C. Fothergill, "Electrical Degradation and Breakdown in Polymers", Peter Peregrinus, London, 1992

[3] G. Chen, T. Y. G. Tay, A. E. Davies, Y. Tanaka and T. Tanaka, "Electrodes and Charge Injection in Low-Density Polyethylene using Pulsed Electroacoustic Techniques”, IEEE Trans. Dielectr. Electr. Insul. Vol. 8, pp. 867-873, 2001

[4] T. Hori, K. Kaneko, T. Mizutani and M. Ishioka, "Effect of electrodes on space charge in low-density polyethylene", Proc. 7 ICPADM, Vol. 3,
pp.XSS-858, 2003

[5] K. Matsui, A. Miyawaki, Y. Tanaka, T. Takada, R. Liu and T. Maeno, "Space charge behavior in LDPE with evaporated electrode under ultra-high electric field”, Proc. ISEIM, Vol. 2, pp. 103-106, 2005

[6] Y. Tanaka, K. Mastui, T. Takada and T. Maeno, "Analysis of Packet-like Space Charge Behavior in Low-density Polyethylene”, Proc. 9 ICSD, pp. 482-485, 2007

[7] K. Matsui, Y. Tanaka, T. Takada, T. Fukao, K. Fukunaga, T. Maeno and J. M. Alison, "Space Charge Behavior in Low-density Polyethylene at Pre Breakdown", IEEE Trans. Dielectr. Electr. Insul. Vol. 12, pp. 406-415, 2005

[8] M. Fukuma, K. Fukunaga and T. Maeno, "Space Charge Dynamics in LDPE Film Immediately Before Breakdown”, IEEE Trans. Dielectr. Electr. Insul. Vol. 8, pp. 304-306, 2001

[9] D. Fabiani, G. C Montanari, L. A. Dissado, C. Laurent and G. Teyssedre, "Fast and Slow Charge Packets in Polymeric Materials under DC Stress", IEEE Trans. Dielectr. Electr. Insul. Vol. 16, pp. 241-250, 2009

[10] Y. Li and T. Takada, "Space Charge Distribution in Multi-Ply LDPE", Electrical Insulation and Dielectric Phenomena. Annual Report, Victoria, BC, Canada, pp. 397-402, 1992

[11] Y. Li, T. Takada, H. Miyata and T. Niwa, "Observation of Charge Behavior in Multiply Low-Density Polyethylene", J. Appl. Phys. vol. 74, pp. 2725-2730, 1993

[12] F. Rogti, A. Mekhaldi and C. Laurent, "Space Charge Behavior at Physical Interfaces in Cross-linked Polyethylene under DC Field”, IEEE Trans. Dielectr. Electr. Insul. Vol. 15, pp. 1478-1485, 2008

[13] K. E. Gubbins and N. Quirke, "Industrial Applications of Molecular Modeling”, Gordon and Breach, London, 1996

[14] D. Frenkel and B. Smit, "Understanding Molecular Simulation, Second Edition: From Algorithms to Applications”, Academic Press, London, 2002

[15] M. P. Allen and D. J. Tieldesley, "Computer simulation of liquids", Oxford Science, Bristol, 1994

[16] R. M. Martin, "Electronic Structure: Basic Theory and Practical Methods”, Cambridge University Press, 2004

[17] J. P. Perdew, "Density-functional approximation for the correlation energy of the inhomogeneous electron gas”, Phys. Rev. B Vol. 33, pp.88228824, 1986.

[18] P. Hohenberg and W. Kohn, "Inhomogeneous Electron Gas", Phys. Rev. Vol. 136, pp. B864-B871, 1964.

[19]W. Kohn and L. J. Sham, "Self-consistent Equations Including Exchange and Correlation Effect”, Phys. Rev. Vol. 140, pp. A1133-A1138, 1965.

[20] C. Lee, W. Yang and R. G. Parr, "Development of the Colle-Salvetti correlation-energy formula into a functional of the electron density", Phys. Rev. B Vol. 37, pp. 785-789, 1988.

[21] R. Peverati and D. G. Truhlar, "The quest for a universal density functional: The accuracy of density functionals across a broad spectrum of databases in chemistry and physics", Phil. Trans. R. Soc. A pp. 1-80, 2013.

[22] R. Car and M. Parrinello, "Unified Approach for Molecular Dynamics and Density Functional Theory", Phys. Rev. Lett. Vol. 55, pp. 2471-2474, 1985

[23] J. Klimes and A. Michaelides, "Perspective: Advances and challenges in treating van der Waals dispersion forces in density functional theory", $\mathrm{J}$. Chem. Phys. Vol. 137, pp. 120901-120912, 2012.

[24] E. Runge and E. K. U. Gross, "Density-Functional Theory for TimeDependent Systems", Phys. Rev. Lett. Vol. 51 pp. 997-1000, 1984.

[25] M. K. Y. Chan and G. Ceder, "Efficient Band Gap Prediction for Band Gaps”, Phys. Rev. Lett. Vol. 105, pp. 196403-196407, 2010.

[26] F. Tran and P. Blaha, "Accurate Band Gaps of Semiconductors and Insulators with a semi-local Exchange-correlation Potential”, Phys. Rev. Lett. Vol. 102, pp. 226401-226404, 2009.

[27] D. Cubero, N. Quirke and D. F. Coker, "Electronic transport in disordered n -alkanes: From fluid methane to amorphous polyethylene”, J. Chem. Phys. Vol. 119, pp. 2669-2679, 2003.

[28] F. Webster, P. J. Rossky and R. A. Friesner, "Nonadiabatic processes in condensed matter: semi-classical theory and implementation", Comp. Phys. Comm. Vol.63, pp. 492, 1991.

[29] L. Turi and P. J. Rossky, "Theoretical Studies of Spectroscopy and Dynamics of Hydrated Electrons”, Chem. Rev. Vol.112, pp. 5641-5674, 2012.

[30] W. Cheney and D. Kincaid, "Linear Algebra: Theory and Applications, Edition 2, Johns and Barlett Learning, Burlington, 2012.

[31] M. Barbatti, "Nonadiabatic dynamics with trajectory surface hopping method”, Comput. Mol. Sci. Vol.1, pp. 620-633, 2011.

[32] J. C. Tully, "Classical and Quantum Dynamics in Condensed Phase Simulations”, World Scientific, Singapore, 1998. 
[33] J. C. Tully, "Perspective: Nonadiabatic dynamics theory", J. Chem. Phys. Vol. 137, pp. 22A301, 2012.

[34] D. Rigby and R. J. Roe, "Molecular dynamics simulations of polymer liquid and glass. I. Glass Transition”, J. Chem. Phys. Vol. 87, pp.7285, 1987.

[35] D. Rigby and R. J. Roe, "Molecular dynamics simulations of polymer liquid and glass. II. Short range order and orientation correlation", J. Chem. Phys. Vol. 89, pp.5280, 1987.

[36] D. Rigby and R. J. Roe, "Molecular dynamics simulations of polymer liquid and glass. 3. Chain information”, Macromolecules, Vol. 22, pp. 22592264, 1989.

[37] D. Rigby and R. J. Roe, "Molecular dynamics simulations of polymer liquid and glass. 4. Free-volume distribution”, Macromolecules, Vol. 23, pp. 5312-5319, 1990.

[38] I. Zuniga, I. Bahar, R. Dodge and W. L Mattice, "Molecular dynamics analysis of transistions between rotational isomers of polyethylene", J. Chem. Phys. Vol. 95, pp. 5348, 1991.

[39] J. J. De Pablo, M. Laso and U. W. Suter, "Simulation of Polyethylene below and above melting point”, J. Chem. Phys. Vol. 96,pp. 2395, 1992.

[40] R. Martonak, W. Paul and K. Binder, "Orthorhombic phase of crystalline polyethylene: A constant pressure path-integral Monte Carlo study", Phys. Rev. E. Vol. 57, pp. 2425-2437, 1998.

[41] T. A. Kavassalis and P. R. Sundararajan, "A molecular-dynamics study of polyethylene crystallization”, Macromolecules, Vol. 26, pp.4144-4150, 1993.

[42] P. R. Sundararajan and T. A. Kavassalis, "Molecular Dynamics Study of Polyethylene Chain Folding: The Effects of Chain Length and the Torsiaonal Barrier”, J. Chem. Phys. Vol. 91, pp. 2541-2549, 1995.

[43] G. Ungar, J. Stejny, A. Kelly, A. I. Bidd and M. C. Whiting, "The crystallization of ultralong normal paraffins: the onset of chain folding", Science, Vol. 229, pp. 368,1985

[44] Y. Wang, D. Mackernan, D. Cubero, D. Coker and N. Quirke, submitted [45]Y. Wang, K. Wu, D. Cubero, D. Mackernan, D. Coker and N. Quirke, "Electron Trapping in Polyethylene", Proc. 13 ICSD, pp.9-13, 2013.

[46] Accelrys 2012

[47]S. Plimpton, "Fast Parallel Algorithms for Short-Range Molecular Dynamics”, J. Comp. Phys. Vol. 117, pp. 1-19, 1995. See also http://lammps.sandia.gov

[48] B. Smit, S. Karaborni and J. I. Siepmann, "Computer simulations of vapour-liquid phase equilibria of n-alkanes”, J. Chem. Phys. Vol. 102, pp. 2126-2140, 1995.

[49] G. Dlubek, T. Lupke, H. M. Fretwell, M. A. Alam, A. Wutzler and H. J. Radusch, "The local free volume in deformed polyethylene: a postron life time study”, Acta Polymer, Vol. 49, pp. 236-243, 1998.

[50] http://www.surface-tension.de/solid-surface-energy.htm

[51] M. C. Righi, S. Scandolo, S. Serra, S. Iarlori, E. Tosatti and G. Santoro,

"Surface States and Negative Electron Affinity in Polyethylene", Phys. Rev. Lett. Vol. 87, pp. 076802, 2001.

[52] S. Serra , S. Iarlori, E. Tosatti, S. Scandolo , G. E. Santoro , "Selftrapping vs. non-trapping of electrons and holes in organic insulators: polyethylene”, Chem. Phys. Lett. Vol.360, pp. 487-493, 2002.

[53] D. Ceresoli, M. C. Righi, E. Tosatti, S. Scandolo, G. Santoro and S Serra, "Exciton self-trapping in bulk polyethylene", J. Phys.: Condens. Matter. Vol. 17, pp. 4621-4627, 2005.

[54] C. R. Bealing and R. Ramprasad, "An atomistic description of the highfield degradation of dielectric polyethylene”, J. Chem. Phys. Vol. 139, pp. 174904, 2013.

[55] M. Meunier and N. Quirke , "Molecular modeling of electron trapping in polymer insulators”, J. Chem. Phys. Vol.113, pp. 369-375, 2000.

[56] DMOL, "DMOL User Guide”, Molecular Simulations Inc. San Diego, 1997.

[57] M. Meunier, N. Quirke and A. Aslanides, "Molecular modeling of electron traps in polymer insulators: Chemical defects and impurities", J. Chem. Phys. Vol. 115, pp. 2876-2881, 2001.

[58] D. Ceresolia, E. Tosatti, S. Scandolo, G. Santoro and S. Serra, "Trapping of excitons at chemical defects in polyethylene”, J. Chem. Phys. Vol. 121, pp. 6478-6484, 2004.

[59] A. Huzayyin, S. Boggs and R. Ramprasad, "Density Functinal Analysis of Chemical Impurities in Dielectric Polyethylene”, IEEE Trans. Dielectr. Electr. Insul. Vol. 17, pp. 926-930, 2010.

[60] D. Cubero, N. Quirke and D. F. Coker, "Electronic states for excess electrons in polyethylene compared to long-chain alkanes", Chem. Phys. Lett. Vol. 370, pp. 21-25, 2003.

[61] P. W. Anderson, "Absence of Diffusion in Certain Random Lattice", Phys. Rev. Vol. 109, pp. 1492-1505, 1958.
[62] D. Mahan, “Many-Particle Physics”, Plenum, New York, 1999.

[63] D. Cubero and N.Quirke, "Computer simulations of localized small polarons in amorphous polyethylene”, J. Chem. Phys. Vol. 120, pp. 77727778, 2004

[64] J. C. Tully and R. K. Preston, “Trajectory Surface Hopping Approach to Nonadiabatic Molecular Collisions: The Reaction of H+ with D2", J. Chem. Phys. Vol. 55, pp. 562-572, 1971.

[65] J. A. Anta, G. Marcelli, M. Meunier and N. Quirke, "Models of Electron Trapping and Transport in Polyethylene: Current - Voltage Characteristics", J. Appl. Phys. Vol. 92, pp. 1002-1008, 2002

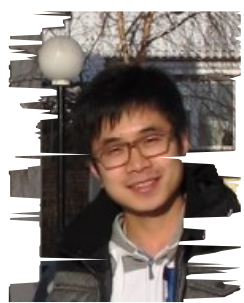

Yang Wang was born in Jilin, China, in 1984. He received the B.S. degree in applied physics from Xi'an Jiaotong University, Xi'an, China, in 2007. Now, he is a Ph.D student in the Department of Electrical Engineering in Xi'an Jiaotong University. During his PhD study from 2010 to 2012, he was in University College Dublin for a joint cultivating program focusing his research on molecular simulation on electronic states in polyethylene.

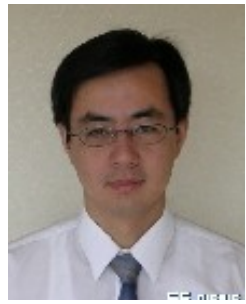

Kai Wu (M’00) was born in China in 1969. He received the M.S. and Ph.D. degrees in electrical engineering from Xi'an Jiaotong University, China in 1992 and 1998, respectively. He was a postdoctoral fellow from 1998 to 2000 and then joined the staff from 2000 to 2003 at Nagoya University, Japan. In 2003, he worked as a research associate at the University of Leicester, UK. In 2004 and 2005, he was a visiting researcher at the Central Research Institute of Electric Power Industry, Japan. Since 2006, he has become a professor of Xi'an Jiaotong University, China.

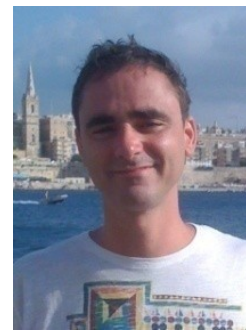

David Cubero was born in Spain in 1971. He received his Ph.D degree in physics from University of Seville in 1996. He was a post-doctorate researcher in Imperial College London, UK, from 2001 to 2004. He is now an associate professor at the University of Seville (Spain) specializing in the area of nonequilibrium statistical physics, including transport theory and resonant phenomema. He has published around 30 papers in leading journals on a wide range of topics in Stastistical Mechanics, including granular flows, Brownian motors, relativistic systems, and electronic transport.

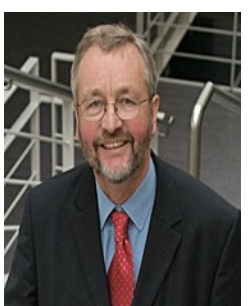

Nick Quirke was born in London,UK, in 1952. He received his B.S. and Ph.D degree in physics from University of Leicester in 1973 and 1977 respectively. He is now a Professor of Chemical Physics at Imperial College, London. His group conducts theoretical research in the general area of nanomaterials with particular interest in their interaction with biological interfaces and polymers. He is a Fellow of the Royal Society of Chemistry, and the Institute of Nanotechnology, Editor-in-Chief of the international Journals, Molecular Simulation, and the Journal of Experimental Nanoscience. He received the 1998 Royal Society of Chemistry Medal for Thermodynamics and was Royal Society / Kan Tong Po Professor at the University of Hong Kong for 2003/2004. He was awarded the 2006 NSTI Fellow Award for Outstanding Contributions in Advancing Nanotechnology and in 2013 a Yangtze river fellowship at Xi'an Jiaotong University. His career has included leadership positions in both academia and industry. He was Principal Research Associate at BP. Most recently he was Vice President and Principal of engineering and science, University College Dublin. 
